Revue belge de géographie

Modelling and benchmarking of borders

\title{
La frontière et son double. Un modèle à partir de l'expérience européenne
}

The border and its double. A model from the European experience

\section{François Moullé}

\section{(2) OpenEdition}

\section{Journals}

\section{Édition électronique}

URL : http://journals.openedition.org/belgeo/10620

DOI : 10.4000/belgeo.10620

ISSN : 2294-9135

Éditeur :

National Committee of Geography of Belgium, Société Royale Belge de Géographie

Référence électronique

François Moullé, «La frontière et son double. Un modèle à partir de l'expérience européenne », Belgeo [En ligne], 1 | 2013, mis en ligne le 31 octobre 2013, consulté le 19 avril 2019. URL : http:// journals.openedition.org/belgeo/10620; DOI : 10.4000/belgeo.10620

Ce document a été généré automatiquement le 19 avril 2019.

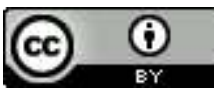

Belgeo est mis à disposition selon les termes de la licence Creative Commons Attribution 4.0 International. 


\title{
La frontière et son double. Un modèle à partir de l'expérience européenne
}

The border and its double. A model from the European experience

\author{
François Moullé
}

\section{Introduction}

1 Depuis plus de quinze ans, l'Europe a vécu des mutations avec la mise en place d'un espace de libre circulation des personnes. Alors que les frontières internes de l'Europe ont été dévaluées, des enclaves spatiales difficiles d'accès ont émergé, tant pour protéger des zones économiques spécifiques, que des espaces privés liés à l'habitat.

2 Les discontinuités spatiales, constitutives des frontières et des enclavements, révèlent des représentations contribuant à la construction des territoires. Elles se manifestent à travers les médias, les discours, les cartes mentales, les productions artistiques...leurs diversités constituant une matière riche, essentielle à la compréhension des ressentis d'une frontière, d'une discontinuité spatiale en général. Ces représentations se construisent à partir de l'expérience propre du passage, autant que par les perceptions véhiculées par autrui.

3 Face à l'immensité du monde, des limites nettes, clairement définies seront perçues, vécues en tant que protection. A quelles échelles les individus et les groupes trouvent-ils des limites permettant un équilibre entre la fermeture protectrice et l'ouverture nécessaire aux échanges? Selon le positionnement géographique face à la limite, quelles représentations apparaissent? A partir d'une série de points de vue subjectifs, un modèle objectif peut-il être mis en place?

$4 \quad$ Un premier temps va nous permettre de préciser les concepts utilisés dans le champ des études frontalières, de la géographie sociale et de la psychologie de l'espace. 
5 La seconde partie permettra de développer les dynamiques d'ouvertures et de fermetures spatiales en Europe. Enfin, nous chercherons à poser les bases d'un modèle de lecture spatiale pour comprendre les tensions scalaires des discontinuités.

\section{Frontière et discontinuités, filtres et représentations}

6 La coupure spatiale représente des réalités diverses, Jean-Pierre Renard (2002), parmi d'autres auteurs, a cherché à les différencier. "La limite circonscrit deux espaces en soulignant des différences. La discontinuité engendre des processus d'organisation ou de désorganisation de l'espace. La frontière exprime ou révèle des conflits de pouvoirs et de contrôle territoriaux" (Renard, ib.). La discontinuité (Brunet, 1968) est généralement identifiable par l'analyse spatiale où l'organisation de l'espace est objectivable. Par contre l'observation de réalités géographiques intègre une dimension plus subjective où une limite pour les uns ne l'est peut-être pas pour d'autres; il en est de même pour le degré organisationnel de la discontinuité. Cette dernière peut évoluer en frontière si elle devient un enjeu de pouvoir; il y a donc des métamorphoses possibles dans le temps y compris dans son interprétation (Newman, 2006). Ces définitions peuvent s'appliquer à différentes échelles, du local au mondial. L'approche du Groupe frontière (2004) en réaction à une proposition de définition formulée par Jacques Levy (2003) permet une acception large et polysémique de la frontière. Il y a frontière dès lors qu'elle révèle des enjeux de compétences et de souveraineté. La morphologie même de la frontière a évolué et est devenue multiple, intégrant les formes spatiales modernes liées aux mobilités, pour devenir même réticulaire lorsque le mouvement l'emporte sur la fixité de la ligne. Nous parlerons dans le développement de frontière lorsqu'il s'agit du contact entre deux pouvoirs politiques. Le terme de discontinuité sera utilisé là où les enjeux organisationnels l'emportent, lorsque des marqueurs spatiaux différencient nettement deux espaces fonctionnels, comme par exemple, entre espace public et espace privé. Néanmoins, la définition de la frontière est à compléter, d'autant que certaines qualités révélées par la recherche sont transposables à la compréhension de la discontinuité.

Parmi les quatre fonctions proposées par le Groupe frontière (2004), dans son travail de définition, nous passons rapidement sur la frontière comme lieu privilégié d'affirmation et de reconnaissance de pouvoirs politiques, puisque cela corrobore l'approche générique précédente. Les trois fonctions suivantes nous intéressent particulièrement pour mieux comprendre les représentations de la frontière à l'échelle de l'individu.

8 La première fonction est celle de la frontière conçue comme un système de contrôle des flux destinés à assurer une maîtrise du territoire à travers un filtrage. La frontière comme filtre est déterminante; l'acte du passage de la frontière, participe à sa représentation ouverte, sélective, ou fermée. Cela n'exclue pas d'autres fonctions de la frontière, mais elle permet de se concentrer sur les conséquences socio-psychologiques de l'acte, réalisé ou non, du passage. Cela correspond à ce que Michel Lussault (2012) nomme la tans-spatialité. Le contrôle au passage et la surveillance de la ligne représentent les mises en pratique de règles qui constituent les filtres.

9 La présence des filtres participe à la représentation de la frontière comme élément favorisant ou non la protection de ceux qui sont à l'intérieur. Notre question de départ lie le flux, l'acte du passage et les représentations. 
10 territoire (Di Méo, 1998), correspondant à la troisième fonction de la frontière comme construction territoriale qui "met de la distance dans la proximité" (Groupe Frontière, 2004; Arbaret-Schulz, 2008). La psychologie de l'espace alimentée par le modèle proposé par Abraham Moles $(1972,1999)$ est importante. Pour l'individu, l'espace oppose ce qui est proche de ce qui est lointain avec une gradation distinguant ce qui est approprié de ce qui est commun avec une série de coquilles, ou sphères spatiales (Lussault, 2012, p. 68), enveloppes protectrices, de la mieux maitrisée avec la sphère du geste personnel au plus lointain ne relevant pas de l'expérience personnelle de l'espace. Les coquilles les plus appropriées sont celles du geste personnel (la peau), la pièce, le logement, les parties communes de la résidence. Au-delà - la rue, le quartier et la ville - l'appropriation est à la fois individuelle et collective avec une dilution progressive de l'individu dans les représentations communes à un groupe. A. Moles donne une dernière coquille (Moles, Rohmer, 1998, p. 107) avec l'Etat. Nous interprétons ce dernier dans sa dimension territoriale où la frontière constitue à la fois la coquille et les limites du pouvoir de l'Etat. Dans le modèle "molesien", nous nous concentrons sur la frontière de l'Etat et la limite entre espace privé et espace public. Ces deux niveaux constituent des seuils déterminants entre l'espace totalement approprié et l'espace public auquel l'individu fait partie, et celui, au-delà de la frontière d'Etat, qui est étranger. Les coquilles ont la double fonction de distinguer ce qui est à l'intérieur, de ce qui est à l'extérieur et de créer le sentiment de la protection, celui qui rassure face au monde extérieur. La distinction entre proche et lointain renvoie aussi à l'expérience de l'espace que nous retrouvons en géographie sociale avec l'espace vécu (Frémont, 1999) qui se distingue de l'espace perçu.

11 une distinction par l'appartenance matérielle et symbolique à une entité territoriale dont elle est l'expression. Les travaux d'Anssi Paasi (1996) sur la frontière finno-russe montrent l'importance des appartenances au moment où la frontière politique permet une ouverture sur l'autre territoire. Les bouleversements géopolitiques imposent la réévaluation des finalités de la frontière, les dimensions matérielles et symboliques du territoire deviennent centrales pour se distinguer de l'au-delà de la frontière dans un contexte d'ouverture.

Globalement, la dématérialisation de certaines frontières entraine le renforcement de la frontière symbolique.

13 A partir de cette première partie, nous pouvons émettre un double postulat qui assoit la suite de notre démonstration. D'une part, les frontières et discontinuités participent à la construction de l'individu en lui donnant un cadre structurant et protecteur face à l'immensité du monde dont la connaissance est faite de représentations imparfaites, imperfections amplifiées par l'éloignement. D'autre part, l'instabilité accrue du monde depuis les années quatre-vingts liée à l'avènement d'une nouvelle mondialisation (Grataloup, 2010) caractérisée par une augmentation sans précédent des échanges, complexifie la compréhension du monde et de ses représentations. Cette évolution donne à la frontière une fonction symbolique d'autant plus puissante qu'elle est un élément stabilisateur dans un monde où la dématérialisation des échanges s'accélère et les changements d'échelles des enjeux politiques se multiplient.

Belgeo, 1 | 2013 


\section{L'expérience du passage en Europe a fortement transformé les représentations des discontinuités} échelles dans la logique du modèle "molesien". L'étude porte donc prioritairement sur des exemples qui ont une tendance à la fermeture pour le passage des personnes, éléments nécessaires à notre démonstration: frontières de l'espace Schengen et discontinuités des espaces privatifs. Par fermeture, nous entendons ici l'utilisation de filtres réglementaires et physiques pour le passage des personnes. Les filtres définissent celles dont le passage est souhaité et celles dont le passage est refusé. Face à la dévaluation des frontières intérieures de l'Europe - Debordering (Scott, 2009) - nous assistons à des phénomènes de renforcement - Rebordering - au moins partiellement. politiques des Etats européens, a pour objectif la libre circulation des marchandises, des informations, des capitaux et des personnes. L'innovation, vis-à-vis des frontières des Etats résidait initialement dans l'ouverture des frontières en structurant une évolution des règles douanières favorisant le développement des flux. Les Accords de Schengen en 1985 ont permis la mise en œuvre du Grand marché unique décidée avec l'Acte unique européen de 1986. Schengen est une Convention intergouvernementale qui fixe la volonté politique d'une ouverture des frontières entre les Etats signataires aux flux de personnes. A l'échelle des Etats et de l'Europe, ce sont les conséquences de la mise en place de l'espace Schengen regroupant qui nous intéressent. Les nouveaux Etats membres de l'Union européenne depuis le traité de Maastricht s'engagent à converger vers les règles Schengen. Par ailleurs, un pays comme la Suisse adhére de manière contractuelle à Schengen. Les différents découpages révèlent ainsi la complexité de la différenciation des frontières de l'Europe. entre les pays membres, situés à l'intérieur. Inédite car l'intégration va jusqu'au démantèlement des postes de douanes, marqueurs spatiaux les plus forts du contrôle de la frontière. Les chemins des douaniers, les passages des contrebandiers, les bornes frontières et les panneaux indicateurs, constituent les vestiges du passage. Cette évolution a permis à de nombreux auteurs de parler de frontière post-westphalienne où l'évolution de la frontière permet des opportunités nouvelles en matière de coopération Debordering - (Scott, 2009), favorisant une lente intégration des territoires par le tissage de liens de plus en plus forts. Les collectivités locales œuvrent depuis longtemps pour tisser des liens transfrontaliers de plus en structurant (Moullé, 2007). Curieusement, la téléphonie mobile, est le principal rappel d'une frontière dévaluée. L'opérateur prévient son client, au moment du passage, pour l'assurer de son accompagnement au-delà de la frontière. Le réseau immatériel est devenu un indicateur plus efficace dans le suivi du passage que les moyens mis à la disposition de la puissance publique ne permettent pas.

La deuxième conséquence découle de la première. Pour garantir la libre circulation des personnes dans un espace défini, il faut que l'enveloppe extérieure garantisse le respect de cette liberté en empêchant la pénétration des personnes non-souhaitées. Ainsi, des filtres puissants ont été établis comme, par exemple, un système d'information permettant à l'ensemble des consulats des pays membres d'échanger les données des personnes faisant une demande de visa. Ce même système est à la disposition des douanes

Belgeo, 1 | 2013 
qui ont été renforcées pour garantir un contrôle effectif et systématique sur les lieux de passages vers l'extérieur, les frontières points (ports, aéroports, douanes terrestres). Enfin des moyens de surveillance modernes ont été disposés le long des frontières terrestres et sur les frontières maritimes stratégiques que sont les détroits. Ainsi, l'Europe a vu ses frontières se murer à l'aide de techniques et matériaux contemporains : radars, capteurs, ponctuellement grillages et barbelés (Novosselof, Neisse, 2007) telles les enclaves espagnoles au Maroc (Ceuta et Melilla).

Nous assistons donc à l'émergence d'un système de frontières supranationales permettant à la fois le debordering et le rebordering (Scott, 2009), ou le confirming ne contredit pas le transcending (Dimitrovona, 2008).

19 En Europe, la frontière externe de l'espace Schengen est devenue une coquille essentielle et pourtant ambiguë, complément des frontières internes dévaluées. Ambiguë, elle est lointaine et sa cartographie est elle-même relativement floue puisque peu d'Européens sont capables de donner la liste des pays membres, encore moins nombreux sont ceux capables de la dessiner sur un fond de carte ${ }^{1}$. Les représentations de cette frontière sont liées à des reportages sur les tentatives de passages clandestins et les images de corps sans vie. Sans faire de généralisation ${ }^{2}$, les pays directement concernés par un détroit stratégique diffusent plus régulièrement des reportages dans les journaux d'informations comme au Portugal, en Espagne et en Italie. Ces représentations sont éminemment ambivalentes avec d'un côté le malaise de voir à nos frontières des réalités humaines difficilement soutenables et de l'autre la satisfaction de voir un système de contrôle présenté comme efficace. Pour une minorité 3 , les ressortissants vivant l'expérience du passage, l'enveloppe externe est très concrète mais vécue spatialement de manière ponctuelle. L'expérience ne participe pas à une représentation mentale de l'espace Schengen et de l'Europe en général, ce qui ne veut pas dire qu'il n'y a pas de représentations mentales.

Pour les ressortissants d'autres Etats non-membres ni de l'espace Schengen, ni des autres Etats de l'Espace Economique Européen ${ }^{4}$, la réalité de la frontière externe est radicalement différente. L'obtention d'un visa Schengen est obligatoire pour la très grande majorité des ressortissants d'Etats extérieurs. Seuls les ressortissants de neuf Etats ${ }^{5}$ n'ont pas besoin de visa touristique pour un séjour de 90 jours maximum.

21 La confrontation avec la frontière commence par la recherche de l'obtention du visa dans un consulat du pays d'arrivée ou le pays où le demandeur souhaite séjourner le plus longtemps. La démarche est de plus en plus complexe et nécessite l'obtention préalable de nombreux justificatifs. Avoir un visa, c'est pouvoir entrer dans l'espace Schengen donc franchir les contrôles douaniers. Les travaux d'Olivier Clochard (2007) nous montrent de manière remarquable la situation de ceux qui souhaitent passer sans avoir obtenu un visa ou suite à un refus. L'Europe est un eldorado pour une partie du monde même si la crise économique actuelle s'accompagne d'une baisse de la pression aux frontières. Pour les prétendants au passage, le vécu de la frontière est celui de la muraille dont il faut trouver le passage dérobé, la faiblesse, les failles même si elles sont risquées. Les migrants investissent de grosses sommes pour tenter le passage, la réussite du projet de passage est déterminante pour le migrant et aussi pour sa famille, sa communauté d'origine.

La frontière-mur est paradoxalement matérialisée par l'horizontalité de la mer, par l'espace physique, puisque les passages clandestins se font d'abord par la voie maritime, notamment en Méditerranée. L'enjeu est d'arriver à franchir le trait de côte pour pouvoir se cacher, se fondre dans le paysage, au milieu des habitants des grandes villes pour ne 
pas être remarqué. Pour ceux qui se retrouvent à l'intérieur de l'espace Schengen en situation illégale, soit par une entrée clandestine, soit par la péremption de la date d'expiration du visa, la frontière est à nouveau vécue comme un mur, pas celui de la citadelle mais celle de la prison que l'on ne peut pas quitter. Les migrants errants sur les côtes de la Manche à la recherche d'un moyen de passage vers la Grande-Bretagne en sont la démonstration.

L'évolution de la conception de la frontière pour les citoyens européens est la troisième conséquence. L'idée d'une frontière "obstacle" entre les Etats a disparu sans pour autant effacer la perception des différences culturelles, ce sont ces différences qui font frontière. C'est la conscience de la ligne cartographique donc d'une représentation métrique qui est mise en cause par l'évolution juridique, la perception des différences culturelles et identitaires est donc véhiculée par d'autres représentations à commencer par celles des stéréotypes et des différences législatives. La fluidité des mobilités favorisée par l'accélération des moyens de transport, l'unification monétaire et la banalisation d'un espace touristique européen n'altèrent pas l'idée du passage vers l'étranger, c'est l'ambiguïté européenne. Un élément de substitution à la frontière en tant qu'expérience de passage est celui de la sécurité lié aux transports collectifs et notamment aériens. Le contrôle de sécurité, qui n'a rien à voir avec le service des douanes pour les vols intérieurs, représente une puissance publique (même si nous savons qu'elle est déléguée à des sociétés privées) de contrôle physique et de vérifications des identités. Le simple respect des règles énoncées pour le voyage suffit pour que l'expérience du passage du contrôle de sécurité ne soit pas vécue comme un obstacle mais une contrainte sécuritaire admise par tous les voyageurs créant une forme "d'identité commune". L'hypothèse de l'attentat suffit à faire accepter le contrôle. L'expérience du passage de la ligne ne génère plus une représentation structurée et métrique de la frontière mais le passage du seuil de la sécurité maintient, de manière réduite, l'expérience du franchissement. La frontière est devenue plus abstraite, parfois camouflée par un discours sur la disparition des frontières largement véhiculé par les médias à partir de théories discréditées depuis (Ohmae, 1995). Le passage du seuil de la coquille (Moles), permettant de distinguer les espaces entre eux, s'effrite au niveau des frontières intérieures de l'Europe.

La perception de la frontière externe Schengen, directement liée à la concrétisation d'un espace de libre circulation des personnes, est fondamentalement différente dès lors qu'il s'agit de citoyens européens ou extra-européens. Quelle que soit l'origine du regard porté sur la frontière, cette dernière reste un élément central de la structuration de l'espace européen pour les flux de personnes.

Dans le modèle "molesien", nous avons étudié les transformations de la coquille la plus lointaine, celle entre l'espace commun et l'étranger. Tournons-nous maintenant vers les coquilles directement perceptibles par l'expérience de l'individu notamment entre l'espace privé et l'espace public.

26 A l'intérieur de l'Europe, à une échelle plus fine, d'autres formes de fermetures existent, sortes d'enclaves où les personnes autorisées à y pénétrer correspondent à des critères clairement définis. Les grandes entreprises, les espaces industriels, les zones de stockages constituent des enclaves très surveillées par des sociétés privées utilisant des moyens modernes de surveillance à l'image des frontières sécurisées. Les lieux de passages correspondent à des lieux de contrôles où des agents arrêtent le visiteur pour en vérifier l'identité et l'objet du passage. Que ce soit pour les personnes autorisées ou les autres, la discontinuité est toujours visuellement marquée par la technologie et les perceptions 
directement liées à celles de la fermeture, du grillage, du barbelé, de la caméra vidéo. Ces enclaves ne sont pas nouvelles, mais depuis une vingtaine d'années les marqueurs spatiaux de la fermeture se sont multipliés. Cette évolution est à mettre en parallèle avec une acceptation croissante des moyens de contrôles notamment de l'espace public avec des moyens modernes comme les caméras vidéo.

Certaines enclaves ont un filtre de contrôle lié directement au paiement d'un droit d'accès. Les espaces touristiques sont particulièrement concernés comme les parcs à thèmes, les villages-vacances. Notons au passage que ce sont les mêmes sociétés privées qui ont la charge de la surveillance, par exemple le groupe suédois de sécurité Securitas, des lieux de contrôles des frontières et des discontinuités.

Les discontinuités qui vont nous intéresser particulièrement sont celles qui ont le plus évolué depuis une quinzaine d'années, depuis l'instauration de l'enveloppe Schengen pour la liberté de circulation des personnes sans qu'un quelconque lien apparent puisse être tissé, ce sont celles qui distinguent l'espace de l'habitat privé de l'espace public.

La tendance est la sécurisation de l'espace privé. La construction immobilière intègre une séparation de plus en plus visible entre l'extérieur et l'intérieur. Cela correspond à une demande plus ou moins explicite (Billard, 2009) et une offre qui tend en permanence à répondre d'avance aux besoins supposés des habitants. L'exemple de la construction immobilière en France (Billard, 2009) entre 2000 et 2007 souligne une tendance générale. Globalement, les promotions immobilières collectives, les immeubles, sont livrés avec une logique de fermeture affirmée. Le standard dans les grandes villes est le digicode permettant d'accéder à un sas fermé, véritable transition sécurisée entre l'extérieur et l'intérieur. Un vidéophone permet ensuite de contacter le logement souhaité pour entrer concrètement dans l'immeuble. Les équipements de protection se sont largement diversifiés, de la porte verrouillée nous sommes passés au contrôle où l'électronique et le numérique ont pris leurs places, sans parler du marché économique qui l'accompagne (Ballif, Rosière, 2009).

Pour l'habitant, ces outils marquent nettement leur enveloppe protectrice qui semble rassurante et fait partie intégrante des éléments de l'habitat moderne, au même titre que la traditionnelle porte. La sécurisation touche aujourd'hui les copropriétés comme l'habitat individuel, les grandes comme les villes de tailles moyennes.

31 Ces enclaves participent à la perception des différentiations socio-spatiales en les soulignant par des marqueurs spatiaux, même si le principe de l'enclavement concerne toutes les classes sociales, la rencontre est formellement plus difficile par la matérialisation des différences.

Les perceptions des discontinuités les plus significatives en matière de gestion des filtres face aux flux de personnes en Europe correspondent aux frontières externes de l'espace Schengen et aux enclaves internes notamment celles, en plein développement, liées à l'habitat individuel et collectif.

\section{La frontière et son double, pour un modèle de lecture spatiale}

A partir de l'exemple européen, nous observons des marqueurs spatiaux caractérisant une double protection ; l'une lointaine, l'autre à proximité. Les travaux d'Abraham Moles (1972, 2009) en socio-psychologie de l'espace nous apportent des éléments pour 
comprendre le lien qui pourrait exister entre ces enveloppes à des échelles bien différentes. L'individu n'a pas la même conscience de l'espace proche et de l'espace lointain. L'évolution des frontières en Europe, notamment depuis la mise en œuvre (1995) de la Convention de Schengen a profondément bouleversé le rapport à l'espace. L'unité protectrice de la frontière stato-nationale s'est dévaluée par la liberté de passage aux frontières. Les craintes vis-à-vis de l'au-delà de la frontière sont toujours liées aux flux migratoires, parfois assimilés de manière symbolique au risque historique de l'invasion, de l'occupation. Les frontières entre les Européens ne jouent plus le rôle hautement symbolique de la protection, au point que l'idée de disparition de la frontière s'est largement diffusée. Ce rôle a été juridiquement renvoyé à une échelle supérieure avec la création de la frontière externe Schengen, enveloppe réunissant les Etats partenaires.

En premier lieu, la perception spatiale de cette frontière est celle de la distance. Dans le modèle "molesien", cela est une dilution amplifiée dans l'espace commun où les représentations sont liées à des informations de seconde main et non liées à l'expérience directe et individuelle de l'espace. Même si la frontière de contrôle Schengen peut être physiquement proche (gare, aéroport), elle paraît lointaine. En second lieu, la distance ressentie ne permet pas d'être rassuré par l'existence de la fonction protectrice de la frontière. La frontière est passée d'une perception spatiale relativement concrète à une idée abstraite, seulement relayée par des faits liés aux tentatives de passages de clandestins, eux-mêmes symboles conscients ou inconscients des risques liés aux flux migratoires.

L'instabilité de la protection supposée de la frontière liée à une évolution géopolitique générale va se traduire spatialement par l'affirmation de l'espace de proximité comme élément central de la protection de l'individu, donc du bien être.

L'espace privé a été l'objet d'une évolution matérielle en faveur d'une fermeture protectrice, véritable teichopolitique (Ballif, Rossières, 2009) avec des outils de plus en plus sophistiqués. Ces investissements ont un effet compensatoire vis-à-vis d'un espace public qui semble de moins en moins protecteur. Chaque individu avait en tête le rôle de l'Etatprovidence, la frontière et son contrôle par l'Etat faisant partie des éléments rassurants. Paradoxalement, le système de protection mis en place dans l'espace Schengen est aussi efficace voire plus que les règles des douanes antérieures en matière de maîtrise des flux migratoires, mais cette nouvelle approche politique de la frontière n'est pas un projet partagé.

Selon que l'individu se place à l'intérieur ou à l'extérieur d'une coquille, le regard de l'individu sera différent.

Depuis l'extérieur de l'espace Schengen, les coupures de l'espace constituent un système double avec une grande enveloppe, véritable fortification pour protéger un monde considéré comme enviable notamment depuis des pays plus pauvres, nonobstant la crise économique et sociale actuelle. A l'intérieur de cette citadelle européenne, une multitude d'enclaves apparaissent avec des marqueurs spatiaux suffisamment explicites pour comprendre leurs inaccessibilités. Notons au passage que certaines oppositions sociospatiales peuvent produire les mêmes effets. L'individu n'ose pas pénétrer des espaces dont les marqueurs spatiaux ne correspondent pas à ses critères identitaires, l'espace lui est alors étranger.

Le système spatial se compose alors d'enveloppes emboitées, l'une globale et très structurée géopolitiquement, une intermédiaire où les fonctions de contrôles ont 
partiellement disparu au profit d'initiative locale (debordering) difficile à comprendre, des enveloppes ou coquilles locales aux formes plus diversifiées mais toujours conçues pour être un obstacle et un outil de contrôle des flux.

Figure 1. L'Europe vue depuis l'extérieur.

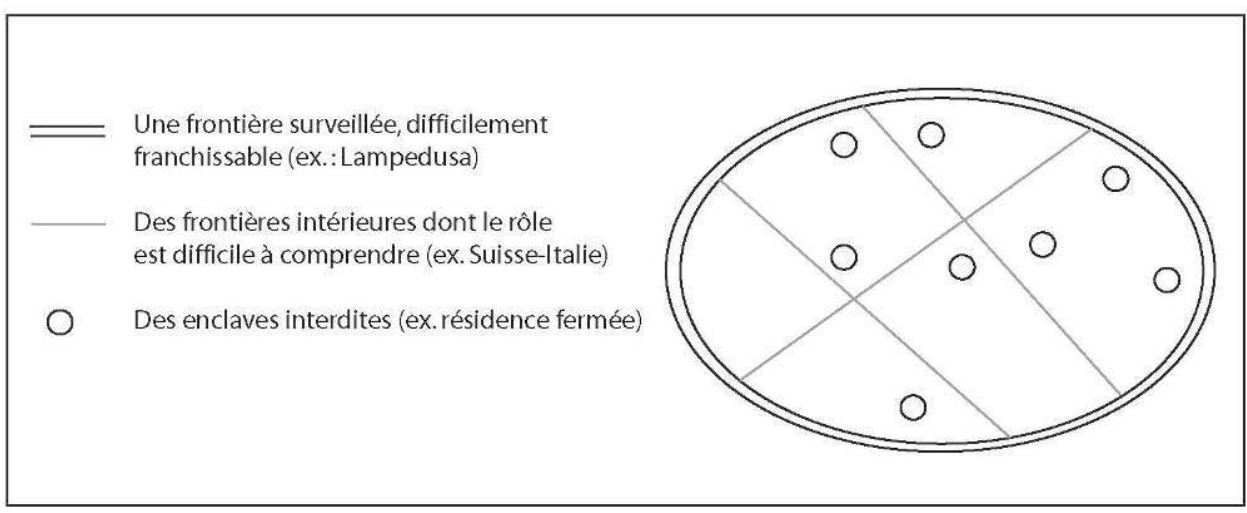

Depuis l'intérieur, la logique est aussi celle de la double enveloppe. Une locale, visible et protectrice où l'on participe directement à la maîtrise du filtre face aux flux extérieurs. L'autre est lointaine, résultat d'un discours sur une efficacité théorique difficile à percevoir. Cette seconde enveloppe n'est ni le fruit de l'expérience directe du passage pour de nombreux européens, ni le résultat d'une série de représentations qui seraient suffisamment solides pour être rassurantes. La distance, l'éloignement de l'enveloppe externe renforce le besoin d'une enveloppe plus proche, une forme de giron dont ont ressent inconsciemment les bénéfices.

Figure 2. L'Europe vue depuis l'intérieur.

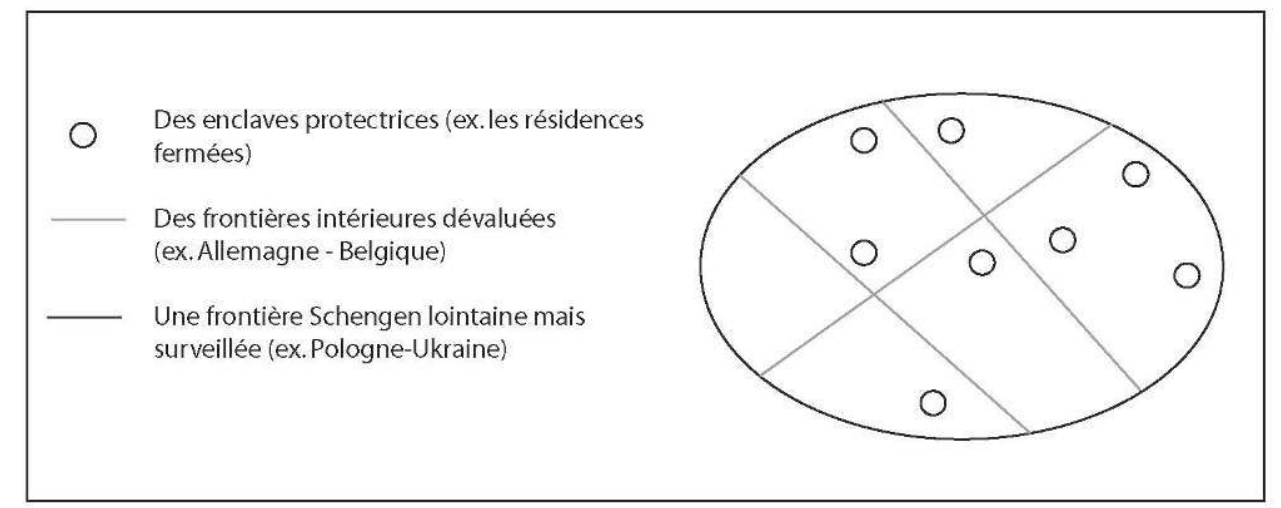

41 Ce modèle de la double enveloppe, représentation de discontinuités et de frontières face aux flux extérieurs, n'est sans doute pas une exclusivité européenne. Partout dans le monde se sont mis en place des quartiers fermés plus connus sous le terme de Gated Communities. L'exemple de la région de Los Angeles est largement connu avec ses multiples quartiers accessibles par des postes de contrôles dont les apparences se rapprochent du poste de douane. Les représentations positives de ces quartiers (Billard, Brennetot, 2009) sont largement partagées par les médias. L'Amérique latine, l'Asie, l'Afrique voient des formes spatiales comparables parfois sous la pression de l'insécurité urbaine notamment nocturne des grandes villes africaines (Houssay-Holzschuch, 2009), le 
plus souvent dans une logique d'une reproduction d'un entre soi considéré comme protecteur.

Dans le modèle de la double enveloppe, les Gated Communities seraient une adaptation à un monde extérieur trop perméable aux flux de personnes. Certes, le modèle américain est comparable à l'Europe actuelle dans le sens où les frontières entre Etats sont relativement dévaluées au moins en terme de gestion des flux, la frontière américaine est cette grande enveloppe qui la sépare du Mexique et du Canada. Ces dyades (Fouchet, 1991, p. 15), à partir de 1992, et surtout suite aux attentats de 2001 ont été sécurisées au point d'être considérées comme des modèles spatiaux (Brunet-Jailly, 2007). La problématique du lien entre le local où l'individu à des représentations liées à ses expériences spatiales, l'espace vécu (Frémont, 1999) et l'immensité de l'espace géopolitique où l'espace perçu devient une construction à partir de matériaux sans liens directs avec l'expérience du contact spatial, doit être posé. Le discours sur l'enveloppe protectrice de la frontière géopolitique n'est sans doute plus suffisant, la protection doit être concrète et matériellement visible, appartenir au quotidien pour ne plus se poser la question de son existence.

Le modèle de la double enveloppe n'implique pas un lien discursif, son observation empirique à travers le monde permet simplement de comprendre que le local a besoin, à son échelle, de marqueurs spatiaux symboliques suffisamment puissants pour rassurer l'individu face à l'impression d'absence de maîtrise sur l'espace public, qu'il n'arrive pas à appréhender comme un espace maîtrisé. De fait, il y a bien co-existence de deux logiques différentes à deux échelles.

Le modèle de la double enveloppe doit aussi être lu à travers le prisme de la mondialisation. En terme de représentation et de flux migratoires, les paradoxes sont multiples entre l'affirmation d'un modèle libéral (Lussault, 2012), des flux matériels et immatériels et les représentations souvent brutales des fermetures où le passage de la frontière est impossible pour certaines personnes. Ce monde où les représentations alternent entre fermetures et ouvertures est déstabilisant. Les frontières, enveloppes géopolitiques, sont devenues des filtres complexes et techniques dont les représentations ne sont elles-mêmes pas faciles à interpréter car toujours multiples. Mettre de la distance dans la proximité (Arbaret-Schultz, 2008) est une réponse locale. La logique de la transspatialité (Lussault, 2012) contrôlée permet un équilibre dans la tension qui oppose ouverture néo-libérale et enfermement par la construction de seuils aux forts pouvoirs filtrants.

De manière plus générale, nous pouvons simplement constater que la mondialisation s'accompagne, d'une part par le développement des quartiers fermés, et de l'autre par le regroupement macro-régional de plusieurs Etats en enveloppes géopolitiques permettant de diminuer les effets des filtres frontaliers entre les pays membres. Le modèle de la double enveloppe, sans parvenir à garantir des causalités, permet au moins de poser des hypothèses d'interprétation des effets des représentations en termes de discontinuités aux différentes échelles.

46 Ce modèle peut être accompagné de l'idée, constatée à l'échelle de la gouvernance métropolitaine transfrontalière (Sohn, Reitel, 2012), du rééchelonnement ou Rescaling (Nelles, Durand, 2012). Les individus, les acteurs publics ne se contentent plus d'échelles d'actions prédéfinies par l'approche institutionnelle des territoires. L'action se réalise à l'échelle qui semble, au moment de sa mise en œuvre, la plus adaptée. Le renforcement de la discontinuité locale entre l'espace public et l'espace privé est une réponse maîtrisée des 
acteurs confrontés aux questions de sécurité locale comme les promoteurs, les propriétaires, les offices de logements sociaux et finalement les responsables politiques territoriaux. Entre la mondialisation et le risque de voir des Etats déstabilisés par l'ouverture de leurs frontières, le macro-régionalisme comme l'Union européenne ou le Mercosur est une réponse pour favoriser les échanges tout en rassurant les acteurs concernés par ces espaces. Le Rescaling est sans doute une stratégie spatiale qui permet de participer à la compréhension du modèle de la double enveloppe dans une période où les enjeux des flux sont à la fois des opportunités et des risques. La mise en place des filtres à certaines échelles dans le cadre de teichopolitiques (Ballif, Rosières, 2009) cherche à maîtriser au mieux ces enjeux.

\section{Conclusion}

Les mutations territoriales en Europe constituent un champ d'investigation particulièrement performant pour analyser les représentations liées aux discontinuités, elles-mêmes en mutations tant à l'échelle des frontières des Etats qu'à l'intérieur des Etats, notamment à l'échelle des villes où la majorité des européens vit. Une discontinuité locale, comme une frontière entre deux Etats, est par essence ressentie de part et d'autre de la ligne. Le modèle "molesien" permet une analyse à différentes échelles, chaque enveloppe est un passage lié à des représentations allant du plus intime au plus général.

La démarche a permis de proposer un modèle de double frontière. La première, lointaine pour la majorité des Européens, est la frontière externe de l'espace Schengen, la seconde, intégrée dans l'espace vécu des individus, est la limite de plus en plus nette entre l'espace public et des espaces inaccessibles, notamment l'espace privé de l'habitat. Nous pouvons parler de la frontière et son double même si les causalités ne sont pas évidentes. Les marqueurs spatiaux se confondent, à tel point que franchir la frontière Schengen ou franchir l'enveloppe de certaines enclaves sont aussi difficiles et porteurs de représentations aussi fortes. Frontières et discontinuités pourraient se confondre, la dimension politique restant l'élément différenciateur.

Ce modèle spatial de la frontière et son double lié aux représentations se retrouve ailleurs dans le monde où les enjeux géopolitiques sont souvent différents. Les connexions sont possibles avec les effets de la mondialisation où le rééchelonnement des stratégies spatiales individuelles et collectives est nécessaire pour s'adapter aux tensions créées entre ouverture et fermeture au monde.

\section{BIBLIOGRAPHIE}

ARBARET-SCHULZ C. (2008), "La question du continu et du discontinu à l'épreuve de la dimension technique des sociétés”, in ALEXANDRE A. et GÉNIN A., Continu et discontinu dans l'espace géographique, Presses Universitaires François Rabelais, pp. 409-416.

BAILLE J. (sous la dir.) (2006), Seuil, du mot au concept, Presse Universitaire de Grenoble, 202 p. 
BALLIF F., ROSIERES S. (2009), "Le défi des teichopolitiques. Analyser la fermeture contemporaine des territoires”, L’Espace Géographique, 38, 3/2009, pp. 193-206.

BILLARD G. et alii (2009), “Typologie et représentations des ensembles résidentiels fermés ou sécurisés en France”, Les nouveaux territoires de la sécurité, Cahiers de la sécurité 9, avril-juin, INHES, pp. 63-73.

BILLARD G., BRENNETOT A., (2009), “Le huis-clos ou l'exaltation du localisme communautaire dans les séries américaines", GRATT On-Line, GRATT Anglophone Studies, 6, pp. 47-70.

BRENNER N. (2001), The limits to scale ? Methodological reflections on scalar structuration, Progress in Human Geography, 25, pp. 591-614.

BRUNET R. (1968), Les phénomènes de discontinuités en géographie, Paris, CNRS, 117 p.

BRUNET-JAILLY E. (2007), Borderlands : Comparing Border Security in North America and Europe, University of Ottawa Press, $406 \mathrm{p}$.

CLOCHARD O. (2007), Le jeu des frontières dans l'accès au statut de réfugié : une géographie des politiques européennes d'asile et d'immigration, Thèse, Poitiers.

DI MEO G. (2001), Géographie sociale et territoires, Nathan, 317 p.

DIMITROVONA B. (2008), “Re-Making of Europe's Borders Throught the European Neighbourhood Policy", Journal of Borderlands Studies, 23, pp. 53-68.

FOUCHER M. (2000), La République européenne, Belin, 147 p.

FREMONT A. (1999), La région, espace vécu, Flammarion, rééd., 288 p.

GAUCHET M. (1985), Le désenchantement du monde, Paris, Gallimard.

GOTTMANN J. (1952), La politique des Etats et leur Géographie, Paris, A. Colin, 228 p.

GRATALOUP C. (2010), Géohistoire de la mondialisation, Le temps long du monde, A. Colin, $2^{\mathrm{e}}$ ed., $288 \mathrm{p}$.

GROUPE FRONTIÈRE, Christiane Arbaret-Schulz, Antoine Beyer, Jean-Luc Piermay, Bernard Reitel, Catherine Selimanovski, Chrsitophe Sohn et Patricia Zander, La frontière, un objet spatial en mutations, EspacesTemps.net, Textuel, 29.10.2004.

HABERMAS J. (1997), L’Espace public : archéologie de la publicité comme dimension constitutive de la société bourgeoise, Payot.

HOUSSAY-HOLZSCHUCH M., VIVET J. (2009), “Blurring the line : sécurisation et 'publicisation' at the Victoria \& Alfred Waterfront”, in BÉNIT-GBAFFOU, SEYI et PEYROUX (dir.), Sécurisation des quartiers et gouvernance locale, enjeux et défis pour les villes africaines, Paris, Karthala-Ifas, pp. 269-289.

LEVY J., LUSSAULT M. (2003), Dictionnaire de la Géographie et de l'espace des sociétés, Belin, $1034 \mathrm{p}$.

LUSSAULT M. (2009), De la lutte des classes à la lutte des places, Grasset, 222 p.

LUSSAULT M. (2012), “Trans-spatialités urbaines”, Revue Hermès, 63, pp. 67-73

MADORE F. et alii (2011), “Quartiers sécurisés : un nouveau défi pour la ville ?”, Carnets de l'Info, $205 \mathrm{p}$.

MOLES A. et ROHMER E. (1972), Psychologie de l'espace, PUF.

MOLES A. et ROHMER E. (2009), Psychologie de l'espace, édition complétée, L'Harmattan, 158 p. 
MOULLE F, 2007, “La coopération transfrontalière institutionnelle dans l'espace", Territoire en Mouvement, 4, pp. 76-81.

MOULLE F. (2010), “Frontières et discontinuités. Regards de géographes”, Revue en ligne Esprit d'avant, octobre, $13 \mathrm{p}$.

NELLES J., DURAND F. (2012), Political rescaling and metropolitan governance in cross-border regions : comparing the cross-border metropolitan areas of Lille and Luxembourg, European Urban and Regional Studies.

NEWMANN D. (2006), "Borders and bordering towards an interdisciplinary dialogue”, European Journal of Social Theory, 9, pp. 171-186

NOVOSSELOF A., NEISSE F. (2007), Des murs entre les hommes, Paris, La Documentation Française, 211 p.

OHMAE K. (1995), The End of the Nation State : The Rise of Regional Economies, Free Press, 214 p.

PAASI A. (1996), Territories, boundaries and consciousness : the changing geographies of the Finnish-Russian Boundary, J. Wiley \& Sons, 353 p.

RENARD J.P. (2002), "La frontière : limite géopolitique majeure mais aussi aire de transition", Limites et discontinuités en géographie, DIEM, SEDES, pp. 40-66.

SCOTT J-W. (2009), "Bordering and Ordering the European Neighbourhood : A critical perspectives on EU Territoriality ans Geopolitics”, Trames, 13, pp. 232-247.

SOHNE Ch., REITEL B. (2012), Le rôle des Etats dans la construction des régions métropolitaines transfrontalières en Europe. Une approche scalaire, CEPS/INSTEAD, coll. Working Papers 2012-42, 24 p.

\section{NOTES}

1. L'exercice a été demandé à 200 étudiants d'histoire de première année, octobre 2010 et 2011. Sur un fond de carte des Etats européens, ils devaient dessiner les limites extérieures de l'espace Schengen. Moins de $10 \%$ des étudiants ont été capables de donner une carte exacte et actualisée (Université d'Artois)

2. Nous n'avons pas de données chiffrées.

3. 77 \% des européens passent leurs vacances dans leur propre pays - Source : 49/2011 - Statistics in focus Eurostat

4. Cela correspond à l'Union européenne à 27 + les anciens Etats membres de l'AELE qui n'ont pas adhéré à l'UE (Norvège, Islande, Lichtenstein). Exception faite de la Suisse qui n'a pas signé l'accord EEE, mais des accords bi-latéraux avec Bruxelles et finalement intégré progressivement (2007-2012) l'espace Schengen.

5. Australie, Brésil, Canada, Corée du Sud, Etats-Unis d'Amérique, Japon, Mexique, Singapour, Venezuela - Source MAE 


\section{RÉSUMÉS}

A partir de propositions théoriques et l'étude des perceptions des frontières et discontinuités en Europe par les Européens et par ceux qui souhaitent y pénétrer, l'auteur propose un modèle de double frontière où des enclaves locales apparaissent pour compenser des frontières politiques nationales considérées comme lointaines et évolutives. Le modèle de la double frontière, extrait de l'expérience européenne, est proposé comme outil de lecture des espaces permettant de comprendre les logiques d'enfermement observables, bien souvent, à deux échelles distinctes. La frontière est analysée dans le sens du passage où les représentations participent au fonctionnement du rôle de filtre face aux flux de population.

From theoretical proposals and study of perceptions of boundaries and discontinuities in Europe by European and by those wishing to enter, the author proposes a model double border where local enclaves appear for compensating political boundaries considered distant and difficult to understand. The model of the double border, extracted from the European experience, is proposed as a tool for reading spaces for understanding the logic of confinement observable, often two different scales. The border is analyzed in the sense of the passage where the representations involved in the running of the role of front filter flows population.

\section{INDEX}

Mots-clés : frontières, discontinuités, représentations, protection, modèle, échelle Keywords : boundaries, discontinuities, model, scale

\section{AUTEUR}

\section{FRANÇOIS MOULLÉ}

Laboratoire DYRT, Université Lille Nord de France, Artois, EA 2468, F- 62000 Arras, France, francois.moulle@univ-artois.fr 